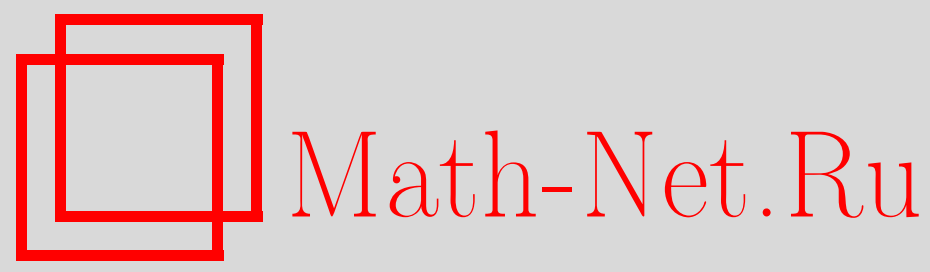

Б. В. Карпов, О структуре жестких полустабильных пучков на алгебраических поверхностях, Матем. заметки, 1998, том 64, выпуск 5, 692-700

DOI: https://doi.org/10.4213/mzm1445

Использование Общероссийского математического портала Math-Net.Ru подразумевает, что вы прочитали и согласны с пользовательским соглашением http://www.mathnet.ru/rus/agreement

Параметры загрузки:

IP: 54.162 .85 .209

26 апреля 2023 г., 03:06:56

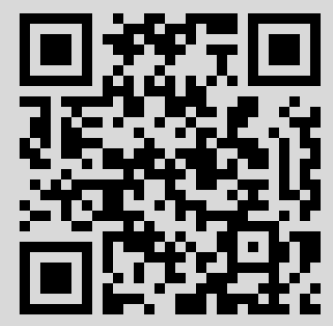


том 64 выпУск 5 нояБРь 1998

УДК 512.723

\section{О СТРУКТУРЕ ЖЕСТКИХ ПОЛУСТАБИЛЬНЫХ ПУЧКОВ НА АЛГЕБРАИЧЕСКИХ ПОВЕРХНОСТЯХ}

Б. В. Карпов

Пусть $S$ - гладкая проективная поверхность, $K$ - ее канонический класс и $H-$ обильный дивизор такой, что $H \cdot K<0$. В статье доказьвается, что для любого жесткого $\left(\operatorname{Ext}^{1}(F, F)=0\right)$ полустабильного по Мамфорду-Такемото относительно $H$ пучка $F$ существует исключительный набор $\left(E_{1}, \ldots, E_{n}\right)$ пучков на $S$ такой, что $F$ может быть построен из $\left\{E_{i}\right\}$ посредством конечного числа расшшрений.

Библиограбфия: 4 названия.

Введение. Пусть $S$ - гладкая проективная поверхность с каноническим классом $K$ и $H$ - обильньй дивизор, для которого $H \cdot K<0$.

Для любого когерентного пучка $F$ на $S$, имеющего $r(F)>0$, рассмотрим следующие инварианты:

$$
\mu(F)=\frac{c_{1}(F) \cdot H}{r(F)} \in \mathbb{Q}
$$

- наклон Мамфорда-Такемото $F$ относительно $H$;

$$
\gamma(F)(n)=\frac{\chi(F(n H))}{r(F)} \in \mathbb{Q}[n]
$$

- наклон Гизекера $F$ относительно $H$.

Когерентньй пучок $F$ без кручения $\mu$-полустабилен ( $\mu$-стабилен), если для любого его подпучка $E$ такого, что $0<r(E)<r(F)$, выполняется неравенство $\mu(E) \leqslant \mu(F)$ $(\mu(E)<\mu(F)$ соответственно). Пучок без кручения $F \gamma$-полустабилен $(\gamma$-стабилен), если для любого подпучка $E \subset F$, имеющего $0<r(E)<r(F)$, неравенство $\gamma(E)(n) \leqslant$ $\gamma(F)(n)(\gamma(E)(n)<\gamma(F)(n)$ соответственно) вьполняется при $n \gg 0$.

Пучок $F$ жесткий, если $\operatorname{Ext}^{1}(F, F)=0$, и сверхжесткий, если $\operatorname{Ext}^{i}(F, F)=0$ при $i>0$. Исключительныи й пучок $E$ - это сверхжесткий пучок такой, что $\operatorname{Hom}(E, E) \cong \mathbb{C}$.

Упорядоченный набор пучков $\left(E_{1}, \ldots, E_{n}\right)$ назьвается исключительным, если все $E_{i}$ исключительны и $\operatorname{Ext}^{i}\left(E_{k}, E_{j}\right)=0$ для любого $i$ при $k>j$.

Цель данной работы состоит в доказательстве следуюшего утверждения.

Работа выполнена при частичной поддержке Российского фонда фундаментальных исследований, грант № 96-01-01323, и фонда INTAS. 
ТЕОРемА. Любой жесткий $\mu$-полустабильный пучок $F$ является сверхжестким и имеет фильтрацию

$$
0=F_{n+1} \subset F_{n} \subset \cdots \subset F_{2} \subset F_{1}=F
$$

все факторь которой $F_{i} / F_{i+1}$ изоморфны $E_{i} \oplus \cdots \oplus E_{i}$ для некоторых исключительных $\gamma$-стабильных пучков $E_{i}$ и набор $\left(E_{1}, \ldots, E_{n}\right)$ исключителен.

Результаты о структуре жестких и сверхжестких пучков тесно связаны с изучением исключительньх наборов пучков на поверхностях дель Пещо (см. [1]) и на поверхностях с численно эффективньм антиканоническим классом (см. [2]). Теорема, сформулированная вьше, является обобщением теоремы 3 из [2, п. 2.4.1].

Конечно, существование обильного дивизора, имеющего отрицательное пересечение с антиканоническим классом, налагает определенные ограничения на поверхность. Однако, основной результат данной работы дает новую информацию, в частности, для рациональных линейчатых поверхностей $\mathbb{P}\left(\mathscr{O}_{\mathbb{P}_{1}} \oplus \mathscr{O}_{\mathbb{P}_{1}}(m)\right), m \geqslant 2$, и для подходящих их раздутий.

Отметим, что для $S=\mathbb{P}\left(\mathscr{O}_{\mathbb{P}_{1}} \oplus \mathscr{O}_{\mathbb{P}_{1}}(m)\right)$ число членов фильтрации жесткого $\mu$-полустабильного пучка $n \leqslant 3$, так как максимальная длина исключительного набора равна $\operatorname{dim} K_{0}(S)=4$ и образы $E_{i}$ в $K_{0}(S)$ лежат в подпространстве $c_{1} \cdot H=r \mu(F)$.

1. Стабильность. Начнем с некоторых обозначений. Мы пишем $\gamma(E)>\gamma(F)$, если $\gamma(E)(n)>\gamma(F)(n)$ при $n \gg 0$. По теореме Римана-Роха

$$
\gamma(F)(n)=\frac{1}{2} n^{2} H^{2}+n\left(\mu(F)+\frac{1}{2} H \cdot K\right)+\frac{\chi(F)}{r(F)} .
$$

Легко видеть, что

$$
\gamma(E)>\gamma(F) \Longleftrightarrow \mu(E)>\mu(F) \text { или } \mu(E)=\mu(F), \quad \frac{\chi(E)}{r(E)}>\frac{\chi(F)}{r(F)} .
$$

Отсюда следует, что любой $\mu$-стабильньй пучок $\gamma$-стабилен и любой $\gamma$-полустабильный пучок $\mu$-полустабилен.

Мы будем использовать следующие свойства стабильных пучков.

Свойство 1. Пучок без кручения $F$ ү-полустабилен ( $\gamma$-стабилен) тогда и только тогда, когда $\gamma(F) \leqslant \gamma(G)(\gamma(F)<\gamma(G)$ соответственно) для любого факторпучка без кручения $G$ пучка $F$.

Свойство 2. Если $E$ u $F \quad \gamma$-стабильны $и \gamma(E)>\gamma(F), \operatorname{mo} \operatorname{Hom}(E, F)=0$.

Свойство 3. Пусть

$$
0 \longrightarrow F^{\prime} \longrightarrow F \longrightarrow G \longrightarrow 0
$$

- точная тройка пучков, где $F$ и $G$-л-полустабильны и $\gamma(F)=\gamma(G)$. Тогда $\gamma\left(F^{\prime}\right)=\gamma(F)$ и $F^{\prime} \quad \gamma$-полустабилен.

Свойство 4. Пусть пучки $E_{1} u E_{2} \quad \gamma$-стабильны, $\gamma\left(E_{1}\right)=\gamma\left(E_{2}\right)$ u $\operatorname{Hom}\left(E_{1}\right.$, $\left.E_{2}\right) \neq 0$. Тогда $E_{1} \cong E_{2} u \operatorname{Hom}\left(E_{1}, E_{2}\right) \cong \mathbb{C}$. 
Свойство 5. Свойства 1-3 справедливы для $\mu$-полустабильных пучков.

Свойства 1-5 доказаны в [2]. Они аналогичны хорошо известным свойствам полустабильных пучков на $\mathbb{P}_{n}$ (см. [3]).

СлЕДСТВИЕ 1. Пусть $E_{1}$ u $E_{2}-\mu$-полустабильные пучки $и \mu\left(E_{1}\right) \geqslant \mu\left(E_{2}\right)$. Тогда $\operatorname{Ext}^{2}\left(E_{2}, E_{1}\right)=0$.

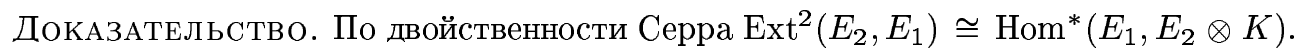
С другой стороны, $c_{1}\left(E_{2} \otimes K\right)=c_{1}\left(E_{2}\right)+r\left(E_{2}\right) K$. Следовательно, мы имеем

$$
\mu\left(E_{2} \otimes K\right)=\mu\left(E_{2}\right)+K \cdot H<\mu\left(E_{2}\right) \leqslant \mu\left(E_{1}\right),
$$

что означает справедливость утверждения в силу свойств стабильности.

2. Свойства фильтраций. Пусть $F-$ пучок. Положим

$$
x F=\underbrace{F \oplus \cdots \oplus F}_{x \text { слагаемых }} .
$$

Для фильтрации

$$
0=F_{n+1} \subset F_{n} \subset \cdots \subset F_{2} \subset F_{1}=F
$$

пучка $F$ обозначим через $\operatorname{Gr}(F)$ упорядоченный набор факторпучков:

$$
\operatorname{Gr}(F)=\left(F_{n} / F_{n+1}, F_{n-1} / F_{n}, \ldots, F_{1} / F_{2}\right) .
$$

Иногда мы будем говорить, что $F$ имеет фильтрацию с $\operatorname{Gr}(F)=\left(G_{n}, G_{n-1}, \ldots, G_{1}\right)$. Это означает, что имеет место фильтрация вида (1) такая, что $G_{i}=F_{i} / F_{i+1}, i=1, \ldots, n$. Отметим, что $G_{n}=F_{n}$.

Мы будем использовать спектральную последовательность

$$
E_{1}^{p, q}=\bigoplus_{i} \operatorname{Ext}^{p+q}\left(G_{i}, G_{i+p}\right),
$$

которая сходится к $\operatorname{Ext}^{p+q}(F, F)$.

Очевидно, что любая фильтрация с двумя факторами есть не что иное как точная тройка

$$
0 \longrightarrow G_{2} \longrightarrow F \longrightarrow G_{1} \longrightarrow 0 .
$$

Лемма МУкаи. Если в точной тройке (3) $\operatorname{Hom}\left(G_{2}, G_{1}\right)=\operatorname{Ext}^{2}\left(G_{1}, G_{2}\right)=0$, $m o \operatorname{dim} \operatorname{Ext}^{1}(F, F) \geqslant \operatorname{dim} \operatorname{Ext}^{1}\left(G_{1}, G_{1}\right)+\operatorname{dim}_{\operatorname{Ext}^{1}}\left(G_{2}, G_{2}\right)$.

Доказательство содержится в [4] и в [1]. Кроме того, это утверждение легко доказать с помощью спектральной последовательности.

ПРЕДЛОЖЕНИЕ 1. Для пучка $F$ следующие условия әквивалентны:

i) существует фильтрация $c \operatorname{Gr}(F)=\left(G_{n}, \ldots, G_{i+1}, G_{i}, \ldots, G_{1}\right)$;

ii) существует фильтрация $\underset{\sim}{c} \operatorname{Gr}(F)=\left(G_{n}, \ldots, G_{i+2}, \widetilde{G}, G_{i-1}, \ldots, G_{1}\right)$, әде $\widetilde{G}$ имеет фильтрацию с $\operatorname{Gr}(\widetilde{G})=\left(G_{i+1}, G_{i}\right)$. 
ДокаЗАТЕЛЬСТво. і) $\Longrightarrow$ ii). Пусть

$$
0 \subset F_{n} \subset \cdots \subset F_{i+2} \subset F_{i+1} \subset F_{i} \subset \cdots \subset F
$$

- фильтрация с $\operatorname{Gr}(F)=\left(G_{n}, \ldots, G_{i+1}, G_{i}, \ldots, G_{1}\right)$. Тогда эта фильтрация с пропущенным $F_{i+1}$ имеет факторы $\operatorname{Gr}(F)=\left(G_{n}, \ldots, G_{i+2}, \widetilde{G}, G_{i-1}, \ldots, G_{1}\right)$. Здесь $\widetilde{G}=F_{i} / F_{i+2}$. Нижняя строка коммутативной диаграммы

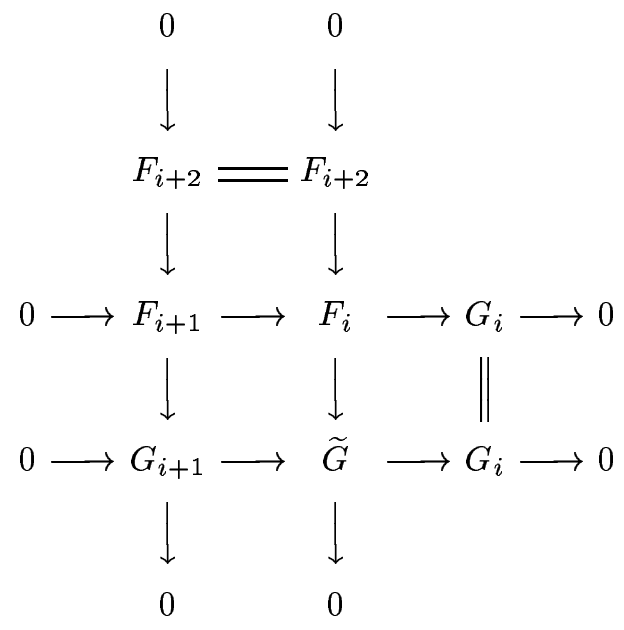

дает фильтрацию пучка $\widetilde{G} \mathrm{c} \operatorname{Gr}(\widetilde{G})=\left(G_{i+1}, G_{i}\right)$.

ii) $\Longrightarrow$ i). В этом случае мы имеем средний столбец и нижнюю строку диаграммы (4). Ясно, что по этим данным остальная часть диаграммы может быть восстановлена, что завершает доказательство.

ПРЕДЛОЖЕНИЕ 2. Пусть имеет место точная тройка пучков

$$
0 \longrightarrow E \longrightarrow F \stackrel{\alpha}{\longrightarrow} \widetilde{F} \longrightarrow 0 .
$$

Тогда следуюшие условия равносильны:

i) $\widetilde{F}$ имеет фильтраиию $c \operatorname{Gr}(\widetilde{F})=\left(G_{n}, \ldots, G_{1}\right)$;

ii) $F$ имеет фильтрацию $c \operatorname{Gr}(F)=\left(E, G_{n}, \ldots, G_{1}\right)$.

ДокАЗАТЕЛЬСТво. Применим индукцию по $n$. Случай $n=1$ очевиден. Рассмотрим случай $n>1$.

i) $\Longrightarrow$ ii). Пусть

$$
0 \subset \widetilde{F}_{n} \subset \cdots \subset \widetilde{F}_{2} \subset \widetilde{F}_{1}=\widetilde{F}
$$

- фильтрация с $\operatorname{Gr}(\widetilde{F})=\left(G_{n}, \ldots, G_{1}\right)$. Тогда $\widetilde{F}_{2}$ имеет фильтрацию с $\operatorname{Gr}\left(\widetilde{F}_{2}\right)=\left(G_{n}, \ldots\right.$, $\left.G_{2}\right)$. Рассмотрим эпиморфизм $F \stackrel{\alpha}{\rightarrow} \widetilde{F} \rightarrow G_{1}$ и его ядро $F_{2}$. Легко видеть, что имеет мес- 
то следующая коммутативная диаграмма:

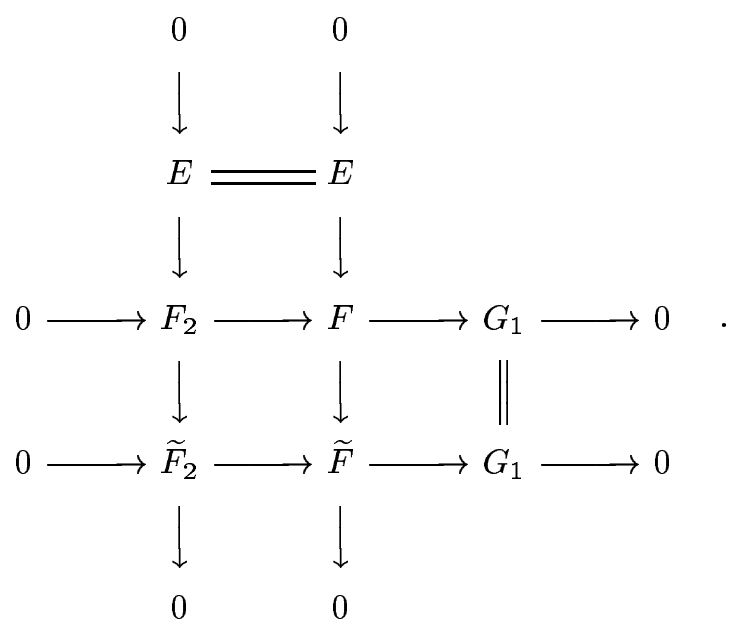

По предположению индукции $F_{2}$ имеет фильтрацию $\mathrm{c} \operatorname{Gr}\left(F_{2}\right)=\left(E, G_{n}, \ldots, G_{2}\right)$. Из средней строки диаграммы (5) вытекает, что $F$ имеет требуемую фильтрацию.

ii) $\Longrightarrow$ i). Пусть

$$
0 \subset E \subset F_{n} \subset \cdots \subset F_{2} \subset F
$$

- фильтрация с $\operatorname{Gr}(F)=\left(E, G_{n}, \ldots, G_{1}\right)$. Тогда мы имеем две верхних строки и средний столбец (5). Ясно, что остальная часть этой диаграммы может быть восстановлена. По индуктивному предположению существует фильтрация $\widetilde{F}_{2}$ с $\operatorname{Gr}\left(\widetilde{F}_{2}\right)=\left(G_{n}, \ldots, G_{2}\right)$. Тогда из нижней строки диаграммы (5) следует, что $\widetilde{F}$ имеет требуемую фильтрацию. Предложение доказано.

Комбинируя предложения 1,2 , легко доказать следуюшее свойство фильтраций.

ПРЕДЛОЖЕНИЕ 3. Следующие утверждения әквивалентны:

i) пучок $F$ имеет фильтрацию $c \operatorname{Gr}(F)=\left(G_{n}, \ldots, G_{1}\right)$, и существуют фильтраиии $\operatorname{Gr}\left(G_{i}\right)=\left(E_{m_{i}}^{(i)}, \ldots, E_{1}^{(i)}\right), i=1, \ldots, n$

ii) пучок $F$ имеет фильтрацию с факторами

$$
\operatorname{Gr}(F)=\left(E_{m_{n}}^{(n)}, \ldots, E_{1}^{(n)}, E_{m_{n-1}}^{(n-1)}, \ldots, E_{1}^{(n-1)}, \ldots, E_{m_{1}}^{(1)}, \ldots, E_{1}^{(1)}\right) .
$$

3. Фильтрации $\gamma$-полустабильного пучка. Всюду в этом пункте мы предполагаем, что пучок $F \quad \gamma$-полустабилен. В работе [2] доказано следующее утверждение.

ПРЕДЛОЖЕНИЕ 4. Пучок F имеет фильтрацию

$$
0=F_{n+1} \subset F_{n} \subset \cdots \subset F_{2} \subset F_{1}=F
$$

с $\gamma$-полустабильными факторами $G_{i}=F_{i} / F_{i+1}$, обладающими свойствами:

а) каждый $G_{i}$ имеет фильтрачию с изоморфными друг другу стабильныцми факторами: $\operatorname{Gr}\left(G_{i}\right)=\left(E_{i}, E_{i}, \ldots, E_{i}\right), \gamma\left(E_{i}\right)=\gamma\left(G_{i}\right)=\gamma(F) ;$

б) $\operatorname{Hom}\left(F_{i}, G_{i-1}\right)=\operatorname{Hom}\left(G_{i}, G_{i-1}\right)=\operatorname{Hom}\left(G_{i-1}, G_{i}\right)=0$. 
Это так называемая фильтрация с изотипными факторами. Она строится по индукции: в качестве $E_{i}$ берется факторпучок пучка $F_{i}$, имеющий $\gamma\left(E_{i}\right)=\gamma(F)$ и минимальный ранг. При этом $E_{i} \gamma$-стабилен и любой ненулевой морфизм $\operatorname{Ker}\left(F_{i} \rightarrow E_{i}\right) \rightarrow E_{i}$ (если такой существует) является эпиморфизмом. Подобным образом $E_{i}$ "отщепляется" от $F_{i}$ до тех пор, пока это возможно, т.е. в результате получается точная тройка $0 \rightarrow F_{i+1} \rightarrow F_{i} \rightarrow G_{i} \rightarrow 0$, где $\operatorname{Gr}\left(G_{i}\right)=\left(E_{i}, E_{i}, \ldots, E_{i}\right)$ и $\operatorname{Hom}\left(F_{i+1}, E_{i}\right)=0$.

ПРЕДЛОЖЕНИЕ 5. Пусть $F$ жесткий. Тогда справедливы следующие утверждения:

a) все $F_{i} u G_{i}$ жесткие;

б) $\operatorname{Ext}^{1}\left(G_{n}, G_{1}\right)=0$;

в) $E_{i}$ исключительны $и G_{i}=E_{i} \oplus \cdots \oplus E_{i}$;

г) $\operatorname{Ext}^{1}\left(E_{n}, E_{i}\right)=0,1 \leqslant i \leqslant n-1$.

ДокАЗАТЕльСТво. а) Пучки $F_{2}$ и $G_{1}$ полустабильны и $\gamma\left(F_{2}\right)=\gamma\left(G_{1}\right)$. Используя следствие 1 , имеем $\operatorname{Ext}^{2}\left(G_{1}, F_{2}\right)=0$. Из леммы Мукаи следует, что $F_{2}$ и $G_{1}$ жесткие. Беря $F_{2}$ в качестве $F$, мы аналогично получаем, что $F_{3}$ и $G_{2}$ жесткие, и т.д.

б) Рассмотрим спектральную последовательность, ассоциированную с фильтрацией пучка $F$. Эта последовательность имеет вид (2) и сходится к $\operatorname{Ext}^{p+q}(F, F)$. Пользуясь следствием 1 , мы получаем $\operatorname{Ext}^{2}\left(G_{i}, G_{j}\right)=0$ для всех $i, j$. Следовательно, первьй член спектральной последовательности имеет вид

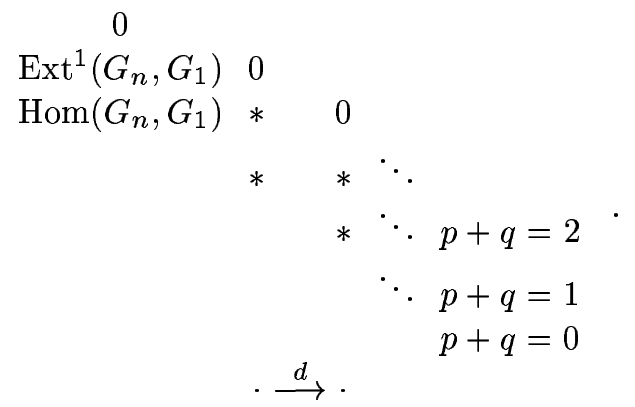

Пучок $F$ жесткий. Значит, спектральная последовательность сходится к 0 на диагонали $p+q=1$. Отсюда $\operatorname{Ext}^{1}\left(G_{n}, G_{1}\right)=0$.

в) Из следствия 1 вытекает, что $\operatorname{Ext}^{2}\left(E_{i}, E_{i}\right)=0$. Беря $G_{i}$ в качестве $F$ в утверждении б), мы получаем $\operatorname{Ext}^{1}\left(E_{i}, E_{i}\right)=0$. Отсюда следует, что $G_{i}=E_{i} \oplus \cdots \oplus E_{i}$. Наконец, $E_{i} \gamma$-стабильны, откуда $\operatorname{Hom}\left(E_{i}, E_{i}\right) \cong \mathbb{C}$.

Утверждение г) следует из в) и б), примененных к $F_{i}$. Предложение доказано.

Таким образом, фильтрация $\gamma$-полустабильного жесткого пучка $F$, описанная в предложениях 4 и 5 , имеет факторы

$$
\operatorname{Gr}(F)=\left(x_{n} E_{n}, \ldots, x_{1} E_{1}\right), \quad \gamma\left(E_{i}\right)=\gamma(F)
$$

где пучки $E_{i}$ исключительны, $\gamma$-стабильны, $E_{i} \not \approx E_{i-1}$ и $\operatorname{Hom}\left(F_{i}, E_{i-1}\right)=0$. 
Предположим, что сушествует $j \in\{1, \ldots, n-1\}$, для которого $\operatorname{Hom}\left(E_{n}, E_{j}\right) \neq 0$. Рассмотрим наибольшее такое $j$. Из свойств стабильности следует, что $E_{j} \cong E_{n}$. Используя утверждение г) предложения 5 и применяя функтор $\operatorname{Hom}\left(E_{n}, \cdot\right)$ к точным тройкам

$$
\begin{aligned}
& 0 \longrightarrow x_{n} E_{n} \longrightarrow F_{n-1} \longrightarrow x_{n-1} E_{n-1} \longrightarrow 0 \\
& 0 \longrightarrow F_{n-1} \longrightarrow F_{n-2} \longrightarrow x_{n-2} E_{n-2} \longrightarrow 0 \\
& 0 \longrightarrow F_{j+1} \longrightarrow F_{j} \longrightarrow x_{j} E_{n} \longrightarrow 0
\end{aligned}
$$

мы получаем $\operatorname{Ext}^{1}\left(E_{n}, F_{n-1}\right)=\operatorname{Ext}^{1}\left(E_{n}, F_{n-2}\right)=\cdots=\operatorname{Ext}^{1}\left(E_{n}, F_{j}\right)=0$. Следовательно, последнее расширение тривиально и $F_{j}=F_{j+1} \oplus x_{j} E_{n}$. Таким образом, мы имеем новую фильтрацию $F$ :

$$
0 \subset\left(x_{n}+x_{j}\right) E_{n} \subset F_{n-1} \oplus x_{j} E_{n} \subset \cdots \subset F_{j+2} \oplus x_{j} E_{n} \subset F_{j} \subset F_{j-1} \subset \cdots \subset F
$$

с факторами

$$
\operatorname{Gr}(F)=\left(\left(x_{n}+x_{j}\right) E_{n}, x_{n-1} E_{n-1}, \ldots, x_{j+1} E_{j+1}, x_{j-1} E_{j-1}, \ldots, x_{1} E_{1}\right) .
$$

Если $E_{j+1} \cong E_{j-1}$, то по предложению 1 мы можем объединить факторы $x_{j+1} E_{j+1}$ и $x_{j-1} E_{j-1}$ в сумму $\left(x_{j+1}+x_{j-1}\right) E_{j-1}$. Соответствуюшая фильтрация - это $(6)$ с пропущенным $F_{j}$. Отметим, что $\operatorname{Hom}\left(F_{i} \oplus x_{j} E_{n}, E_{i-1}\right)=0$ при $i=j+2, \ldots, n$.

Итерируя эту процедуру, мы получаем следующее утверждение.

ПРЕДЛОЖЕНИЕ 6. Существует фильтрация

$$
0 \subset \widetilde{x}_{n} E_{n}=\widetilde{F}_{m+1} \subset \widetilde{F}_{m} \cdots \subset \widetilde{F}_{2} \subset \widetilde{F}_{1}=F
$$

с факторами $\widetilde{\operatorname{Gr}}(F)=\left(\widetilde{x}_{n} E_{n}, \widetilde{x}_{m} \widetilde{E}_{m}, \ldots, \widetilde{x}_{1} \widetilde{E}_{1}\right)$ такими, что упорядоченный набор $\left(\widetilde{E}_{1}, \ldots, \widetilde{E}_{m}\right)$ является поднабором $\left(E_{1}, \ldots, E_{n-1}\right)$ и

$$
\operatorname{Hom}\left(E_{n}, \widetilde{E}_{i}\right)=0, \quad i=1, \ldots, m
$$

Более того, $\widetilde{E}_{m}=E_{n-1}, \operatorname{Hom}\left(\widetilde{F}_{i}, \widetilde{E}_{i-1}\right)=0 u \widetilde{E}_{i} \not \widetilde{E}_{i-1}, 2 \leqslant i \leqslant m$.

4. Доказательство теоремы. Шаг 1. Пучок $F$-полустабилен. Рассмотрим фильтрацию, описанную в п. 3. Она имеет факторы $\operatorname{Gr}(F)=\left(x_{n} E_{n}, \ldots, x_{1} E_{1}\right), \gamma\left(E_{i}\right)=$ $\gamma(F)$, где пучки $E_{i}$ исключительны и $\gamma$-стабильны, $\operatorname{Ext}^{1}\left(E_{n}, E_{i}\right)=0, E_{i} \not E_{i-1}$ и $\operatorname{Hom}\left(F_{i}, E_{i-1}\right)=0$. По предложению 6 мы можем построить эту фильтрацию так, что $\operatorname{Hom}\left(E_{n}, E_{i}\right)=0$ при $i=1, \ldots, n-1$.

Отметим, что в силу следствия $1 \operatorname{Ext}^{2}\left(E_{i}, E_{j}\right)=0$ для всех $i, j$.

Пучок $\widetilde{F}=F / x_{n} E_{n} \gamma$-полустабилен и имеет фильтрацию

$$
0=F_{n} / x_{n} E_{n} \subset F_{n-1} / x_{n} E_{n} \subset \cdots \subset F_{2} / x_{n} E_{n} \subset F_{1} / x_{n} E_{n}=\widetilde{F}
$$

с $\gamma$-полустабильными факторами $\operatorname{Gr}(\widetilde{F})=\left(x_{n-1} E_{n-1}, \ldots, x_{1} E_{1}\right)$. 
Отсюда следует, что $\operatorname{Hom}\left(E_{n}, \widetilde{F}\right)=0$. Из следствия 1 имеем $\operatorname{Ext}^{2}\left(\widetilde{F}, E_{n}\right)=0$. Применяя лемму Мукаи к точной тройке

$$
0 \longrightarrow x_{n} E_{n} \longrightarrow F \longrightarrow \widetilde{F} \longrightarrow 0
$$

заключаем, что $\widetilde{F}$ жесткий.

Применяя функтор $\operatorname{Hom}\left(\cdot, E_{i-1}\right)$ к точной тройке

$$
0 \longrightarrow x_{n} E_{n} \longrightarrow F_{i} \longrightarrow F_{i} / x_{n} E_{n} \longrightarrow 0
$$

получаем, что $\operatorname{Hom}\left(F_{i} / x_{n} E_{n}, E_{i-1}\right)=0$. Следовательно, фильтрация $(7)$ пучка $\widetilde{F}$ обладает свойствами, сформулированными в предложении 4. Тогда из утверждения г) предложения 5 следует, что

$$
\operatorname{Ext}^{1}\left(E_{n-1}, E_{i}\right)=0, \quad i=1, \ldots, n-2 .
$$

Используя предложение 6 , мы можем построить фильтрацию пучка $\widetilde{F}$ с факторами

$$
\operatorname{Gr}(\widetilde{F})=\left(\widetilde{x}_{n-1} E_{n-1}, \widetilde{x}_{m} \widetilde{E}_{m}, \ldots, \widetilde{x}_{1} \widetilde{E}_{1}\right)
$$

такими, что набор $\left(\widetilde{E}_{1}, \ldots, \widetilde{E}_{m}\right)$ является поднабором $\left(E_{1}, \ldots, E_{n-2}\right)$ и

$$
\operatorname{Hom}\left(E_{n-1}, \widetilde{E}_{i}\right)=0, \quad i=1, \ldots, m
$$

(на самом деле, $\widetilde{E}_{m}=E_{n-2}$ ). Из предложения 2 следует, что существует фильтрация пучка $F$ с факторами

$$
\operatorname{Gr}(F)=\left(x_{n} E_{n}, \widetilde{x}_{n-1} E_{n-1}, \widetilde{x}_{m} \widetilde{E}_{m}, \ldots, \widetilde{x}_{1} \widetilde{E}_{1}\right)
$$

Заменяя в предыдущем рассуждении $\widetilde{F}$ на $\widetilde{F} / \widetilde{x}_{n-1} E_{n-1}$ и итерируя эту процедуру, мы получаем фильтрацию $F$ с

$$
\operatorname{Gr}(F)=\left(x_{n} E_{n}, \widetilde{x}_{n-1} E_{n-1}, \widetilde{x}_{n-2} E_{n-2}, x_{k}^{\prime} E_{k}^{\prime}, \ldots, x_{1}^{\prime} E_{1}^{\prime}\right),
$$

где набор $\left(E_{1}^{\prime}, \ldots, E_{k}^{\prime}, E_{n-2}, E_{n-1}, E_{n}\right)$ исключителен.

Шаг 2. Пучок $F \mu$-полустабильный и жесткий. Пусть

$$
0 \subset F_{n} \subset \cdots \subset F_{2} \subset F_{1}=F
$$

- фильтрация Гардера-Нарасимхана $F$ относительно $\gamma($ см. [2]) с факторами $\operatorname{Gr}(F)=$ $\left(G_{n}, \ldots, G_{1}\right)$. Здесь $G_{i} \gamma$-полустабильны и $\gamma\left(G_{i}\right)>\gamma\left(G_{i-1}\right)$. Пучок $G_{n}$ - подпучок $F$ и $G_{1}$ - факторпучок $F$, откуда $\mu\left(G_{n}\right) \leqslant \mu(F) \leqslant \mu\left(G_{1}\right)$, и мы имеем $\mu\left(G_{i}\right)=\mu(F)$. Следовательно, $\mu\left(F_{i}\right)=\mu(F)$ и $F_{i} \mu$-полустабильны.

Покажем, что $G_{i}$ жесткие. Пучок $F_{2}$ имеет фильтрацию с $\operatorname{Gr}\left(F_{2}\right)=\left(G_{n}, \ldots, G_{2}\right)$. По свойствам стабильности $\operatorname{Hom}\left(G_{i}, G_{1}\right)=0, i=2, \ldots, n$. Из этого следует, что $\operatorname{Hom}\left(F_{2}, G_{1}\right)=0$. Согласно следствию 1 имеем $\operatorname{Ext}^{2}\left(G_{1}, F_{2}\right)=0$. Из леммы Мукаи следует, что $G_{1}$ и $F_{2}$ жесткие. Беря $F_{3}$ вместо $F_{2}$, мы аналогично получаем, что $G_{2}$ и $F_{3}$ жесткие. Продолжая рассуждение в том же духе, мы видим, что все $G_{i}$ жесткие. 
По шагу 1 каждьй $G_{i}$ имеет фильтрацию с

$$
\operatorname{Gr}\left(G_{i}\right)=\left(x_{m_{i}}^{(i)} E_{m_{i}}^{(i)}, \ldots, x_{1}^{(i)} E_{1}^{(i)}\right), \quad i=1, \ldots, n
$$

где $E_{s}^{(i)} \gamma$-стабильны, $\gamma\left(E_{s}^{(i)}\right)=\gamma\left(G_{i}\right), s=1, \ldots, m_{i}$, и набор $\left(E_{1}^{(i)}, \ldots, E_{m_{i}}^{(i)}\right)$ исключителен. Из предложения 3 следует, что $F$ имеет фильтрацию с

$$
\operatorname{Gr}(F)=\left(x_{m_{n}}^{(n)} E_{m_{n}}^{(n)}, \ldots, x_{1}^{(n)} E_{1}^{(n)}, \ldots, x_{m_{1}}^{(1)} E_{m_{1}}^{(1)}, \ldots, x_{1}^{(1)} E_{1}^{(1)}\right) .
$$

Мы утверждаем, что набор $\left(E_{1}^{(1)}, \ldots, E_{m_{1}}^{(1)}, \ldots, E_{1}^{(n)}, \ldots, E_{m_{n}}^{(n)}\right)$ исключителен. По построению $\gamma\left(E_{t}^{(j)}\right)>\gamma\left(E_{s}^{(i)}\right)$ при $j>i$, откуда $\operatorname{Hom}\left(E_{t}^{(j)}, E_{s}^{(i)}\right)=0$. С другой стороны, $\mu\left(E_{t}^{(j)}\right)=\mu\left(E_{s}^{(i)}\right)$. Отсюда следует, что $\operatorname{Ext}^{2}\left(E_{t}^{(j)}, E_{s}^{(i)}\right)=0$. Наконец, из спектральной последовательности (2), ассоциированной с фильтрацией $(8)$, вытекает, что $\operatorname{Ext}^{1}\left(E_{t}^{(j)}, E_{s}^{(i)}\right)=0$. Это завершает доказательство.

Автор благодарен С. А. Кулешову за полезные обсуждения, а также А.Н. Рудакову и А. Л. Городенцеву за внимание к этой работе.

\section{СПИСОК ЦИТИРОВАННОЙ ЛИТЕРАТУРЫ}

[1] Кулешов С.А., Орлов Д.О. Исключительные пучки на поверхностях дель Пеццо // Изв. РАН. Сер. матем. 1994. Т. 58. № 3. С. 59-93.

[2] Кулешов С. А. Исключительные и жесткие пучки на поверхностях с антиканоническим классом без базисных компонент. Препринт № 1. М.: Матем. колледж НМУ, 1994.

[3] Оконек К., Шнейдер М., Шпиндлер Х. Векторные расслоения на комплексных проективных пространствах. М.: Мир, 1984.

[4] Mukai S. On the moduli spaces of bundles on K3 surfaces. I // Vector Bundles / ed. Atiyah et al.: Oxford Univ. Press, 1986. P. 67-83. 